\title{
Impact of increasing doses of flavonoid-rich and flavonoid-poor fruits and vegetables on antioxidant status in humans-FLAVURS study
}

\author{
Y. N. Jin, T. W. George, M. F. Chong, A. M. Minihane, M. H. Gordon and J. A. Lovegrove for \\ FLAVURS Study Group \\ Hugh Sinclair Unit of Human Nutrition, Department of Food and Nutritional Sciences, The University of Reading, \\ Whiteknights, PO Box 226, Reading Berks RG6 6AP, UK
}

\begin{abstract}
Emerging epidemiological evidence indicates that the consumption of fruits and vegetables $(\mathrm{F} \& \mathrm{~V})$ is associated with reduced risk of CVD, with evidence that dietary flavonoids present in F\&V may contribute to the CVD benefits of increased F\&V intake. However, a clear dose-response relationship between consumption of F\&V and risk reduction is still absent. The present study investigated the effects of different amounts and types (flavonoid-rich or flavonoid-poor) of $\mathrm{F} \& \mathrm{~V}$ intake on the antioxidant status of human subjects, as an indicator of risk of CVD. In a randomized, controlled parallel intervention study, 154 subjects (ages 30-70 years, 94 females, 60 males) who were at above average risk of developing CVD were randomly recruited into one of the 3 groups: flavonoid-rich F\&V or flavonoidpoor $\mathrm{F} \& \mathrm{~V}$ intervention group or habitual diet group (control). Subjects in the two intervention groups sequentially increased their $\mathrm{F} \& \mathrm{~V}$ intake by 2, 4 and 6 extra portions for 6 weeks across an 18-week period. Blood samples were taken 4 times at week $0,5,11$ and 17 for antioxidant status assessment by plasma ferric reducing antioxidant power (FRAP) and vitamin C concentrations. Both intervention groups showed significantly higher plasma FRAP values than the control group following 4 extra portion $\mathrm{F} \& \mathrm{~V}(P<0.001)$, with the flavonoid-rich group having significantly higher FRAP values than both the flavonoid-poor and the control groups following 6 extra portions F\&V $(P<0.001)$. No significant changes were observed in the FRAP values of the control group throughout the study. The plasma vitamin $\mathrm{C}$ levels of the flavonoid-rich and flavonoid-poor groups were similar and significantly greater than that of the control group following 2,4 and 6 extra portion $\mathrm{F} \& \mathrm{~V}(P=0.001)$. In conclusion, increased consumption of flavonoid-rich or -poor $\mathrm{F} \& \mathrm{~V}$ contributed to significant dose-response increases in vitamin $\mathrm{C}$ and antioxidant status of human subjects.
\end{abstract}

The project was funded by the Food Standards Agency. 